\title{
Non-Gaussian ground-state deformations near a black-hole singularity
}

\author{
Stefan Hofmann 1 , ${ }^{*}$ and Marc Schneider ${ }^{1,0}$ \\ ${ }^{1}$ Arnold Sommerfeld Center for Theoretical Physics, Theresienstraße 37, 80333 München
}

(Dated: April 4, 2017)

\begin{abstract}
The singularity theorem by Hawking and Penrose qualifies Schwarzschild black-holes as geodesic incomplete space-times. Albeit this is a mathematically rigorous statement, it requires an operational framework that allows to probe the space-like singularity via a measurement process. Any such framework necessarily has to be based on quantum theory. As a consequence, the notion of classical completeness needs to be adapted to situations where the only adequate description is in terms of quantum fields in dynamical space-times. It is shown that Schwarzschild black-holes turn out to be complete when probed by self-interacting quantum fields in the ground state and in excited states. The measure for populating quantum fields on hypersurfaces in the vicinity of the blackhole singularity goes to zero towards the singularity. This statement is robust under non-Gaussian deformations of and excitations relative to the ground state. The physical relevance of different completeness concepts for black holes is discussed.
\end{abstract}

PACS numbers: 04.20.Dw, 04.62.+v, 04.70.-s, 11.10.-z

\section{INTRODUCTION}

The singularity theorem [1] by Hawking and Penrose identifies Schwarzschild black-holes as incomplete in a precise sense: Black holes incorporate a space-like singularity where null and time-like geodesics end prematurely, referring to classical point particles that reach these endpoints in a finite time, because their potential is bounded from above [2]. This relates the geometric completeness concept to the usual notion of potential completeness. The latter can be lifted to quantum mechanical completeness, which implies the existence of a unique evolution in compliance with unitarity. Unitarity remains the relevant completeness criterion in static space-times and extends to encompass the relativistic domain of a single particle.

In this context, Horowitz and Marolf [3] were the first to point out that geodesic (and hence potential) incompleteness does not necessarily imply unitarity violation. They gave examples of static space-times with time-like singularities that nevertheless qualified as complete from a quantum mechanical perspective. This was conceptually promising since quantum field theory in a static, globally hyperbolic space-time admits a consistent description of a single relativistic particle, as was shown by Ashtekar and Magnon in [4]. And it was practical, since Wald [5] showed that the dynamics of a Klein-Gordon scalar field in arbitrary static space-times could be examined by asking whether the spatial part of the wave operator admits a self-adjoint extension.

Dynamical space-times in general, however, require a quantum theory with fields as local bookkeeping devices. The interior of Schwarzschild black-holes is a dynamical space-time, even though the exterior is thought of as being static. A strictly unitary evolution is no longer

\footnotetext{
* stefan.hofmann@physik.uni-muenchen.de

$\dagger$ marc.schneider@physik.uni-muenchen.de
}

necessary, even in the absence of interactions. As a consequence, a new criterion for quantum completeness is required that reduces to the classical one (and its quantum mechanical descendant) in appropriate limits, but extends to quantum field theory. While unitarity reflects the symmetry underlying quantum mechanical evolution, the logically more potent concept is state normalisation. Unitarity is replaced by stability, which demands a valid probabilistic interpretation instead of a conserved norm. So stability requires only a semigroup of contractions [ $[6]$. At the intuitive level, stability ensures a probabilistic interpretation of the quantum system in a background dynamical space-time. Such a stability notion clearly reflects on the completeness of the background space-time as scrutinised by quantum fields.

Stability investigations are usually pursued in the asymptotic framework pertinent to scattering theory, which is neither an option in generic space-times, nor is it practical given that instabilities are anchored in regions near classical singularities. In this situation, the Schrödinger representation of quantum field theory turns out to be extremely useful, since it conveniently allows to investigate stability at finite times. Based on this framework, the following completeness criterion [7] has recently been suggested: A globally hyperbolic spacetime is called quantum complete to the left with respect to a free field theory, if its Schrödinger wave functional can be normalised at an initial time $t_{0}$, and if the normalisation is bounded from above by its initial value for all $t \in\left(0, t_{0}\right)$.

In a previous article [7] it has been shown that Schwarzschild black-holes are quantum complete. Here, the notion of quantum completeness is extended to include non-Gaussian deformations of the ground state induced by self-interactions and excited states. As will be shown, all generalisations respect the concept of quantum completeness as suggested above. Concrete calculations are presented for a Schwarzschild black-hole populated by real scalar fields with quartic self-interactions. The wave 
functionals for the ground state as well as for arbitrary excited states are investigated near the black-hole singularity. The main result is that Schwarzschild black-holes are quantum complete even if self-interactions and excitations are permitted. The different completeness concepts employed in physics are logically consistent in their respective domains of validity. Their physical relevance for black-hole interiors is discussed in detail.

\section{GEOMETRIC PRELIMINARIES}

Let us first clarify our conventions. Consider the spacetime $(\mathcal{M}, g)$ with $\mathcal{M}:=\mathbb{R} \times \mathbb{R}^{+} \times S^{2}$, where $S^{2}$ is the unit two-sphere. The projections $t: \mathcal{M} \rightarrow \mathbb{R}$ and $r: \mathcal{M} \rightarrow \mathbb{R}^{+}$ are called Schwarzschild time and Schwarzschild radius, respectively. The Schwarzschild function $h(r):=1-r_{\mathrm{g}} / r$ is increasing from minus infinity at $r=0$ to one as $r$ approaches plus infinity, passing through zero at $r=r_{\mathrm{g}}$. Here, $r_{\mathrm{g}}=2 M$ denotes the gravitational radius of a source of mass $M$. The physical conditions implied by a static and spherical symmetric source in vacuum, supplemented with asymptotic fall-off conditions, give rise to two warped product space-times, the Schwarzschild exterior space-time $\mathcal{E}:=P_{>} \times_{r} S^{2}$, with $P_{>}$denoting the region $r>r_{\mathrm{g}}$ in the $(t, r)$-half plane $\mathbb{R} \times \mathbb{R}^{+}$, and the Schwarzschild black hole $\mathcal{B}:=P_{<} \times_{r} S^{2}$, with $P_{<}$denoting the region $r<r_{\mathrm{g}}$. In $\mathcal{B}$, the coordinate vector field $\partial_{t}$ becomes space-like and $\partial_{r}$ becomes time-like. Owing to this, we write for the metric in $\mathcal{B}$

$$
g=-s^{-1}(t) \mathrm{d} t \otimes \mathrm{d} t+s(t) \mathrm{d} r \otimes \mathrm{d} r+t^{2} w .
$$

Here, $s(t):=\left|1-r_{\mathrm{g}} / t\right|$ and $w$ denotes the metric on $S^{2}$, equipped with the usual spherical coordinates $(\theta, \phi)$.

Spatial hypersurfaces $\Sigma$ in $\mathcal{B}$ are conformally flat as implied by a vanishing Cotton tensor. In order to appreciate conformal flatness, it suffices here to consider the region $t \ll r_{\mathrm{g}}$ close to the space-like singularity of $\mathcal{B}$, where the line element of $P_{<}$takes the approximate form $-\left(t / r_{\mathrm{g}}\right) \mathrm{d} t^{2}+\left(r_{\mathrm{g}} / t\right) \mathrm{d} r^{2}$. Following Ehlers and Kundt [8], and demanding in addition $\theta \ll 1$, the metric can be restated as a type-D Kasner solution characterised by the exponents $\left(p_{1}, p_{2}, p_{3}\right)=(2 / 3,2 / 3,-1 / 3)$. The corresponding coordinate transformation is $r=:\left(3 / 2 r_{\mathrm{g}}\right)^{1 / 3} z$, $t=:\left(9 r_{\mathrm{g}} / 4\right)^{1 / 3} \tau^{2 / 3}$ and $\theta \exp (\mathrm{i} \phi)=:\left(4 / 9 r_{\mathrm{g}}\right)^{1 / 3}(x+\mathrm{i} y)$. In this coordinate neighbourhood, the line element of $\mathcal{B}$ becomes

$$
\mathrm{d} s^{2}=-(\mathrm{d} \tau)^{2}+\tau^{4 / 3}\left((\mathrm{~d} x)^{2}+(\mathrm{d} y)^{2}\right)+\tau^{-2 / 3}(\mathrm{~d} z)^{2} .
$$

Harmonic analysis in $\Sigma$ is similar to Euclidean space. In particular, the Laplace operator in $\Sigma$ factorizes $\Delta_{\Sigma}=$ $g_{a b}(\tau) \partial^{a} \partial^{b}$, with $\tau$ indexing $\Sigma_{\tau}$. Generalised eigenfunctions of $\Delta_{\Sigma}$ are plane waves $\exp \left(\mathrm{i} g_{\Sigma}(k, x)\right)$, where $g_{\Sigma}$ denotes the induced metric tensor in $\Sigma$. This coordinate neighbourhood is useful for a quick examination of our results. Moreover, it allows to relate to the framework suggested by Belinskii, Khalatnikov, and Lifshitz [9]. Let us stress, however, that all the results in this paper have been derived in the usual Schwarzschild neighbourhood.

\section{QUANTUM COMPLETENESS OF SCHWARZSCHILD BLACK HOLES}

In this section, we briefly review the argument showing that $\mathcal{B}$ is quantum complete. The main result is equation (7), which has recently been published in [7], where a considerably more detailed derivation can be found. Subsequently it is shown that the free Hamilton density in the ground state vanishes towards the classically singular hypersurface $\Sigma_{0}$.

Since $\mathcal{B}$ is a globally hyperbolic space-time, it is diffeomorphic to $\mathbb{R} \times \Sigma$, and foliates into spatial hypersurfaces $\Sigma_{t}$ indexed by Schwarzschild time. In $\mathcal{B}$, consider the dynamical system $(\mathcal{H}, \Phi)$, where $\Phi$ denotes a real scalar field with Hamilton density $\mathcal{H}=\mathcal{H}_{\pi}+\mathcal{P}[\Phi]$. Here, $\mathcal{H}_{\pi}=\sqrt{-g_{t t}} / 2 \pi^{2} / \operatorname{det}\left(g_{\Sigma}\right)$ with $\pi=-\mathrm{i} \delta / \delta \Phi$, and $\mathcal{P}[\Phi]$ denotes the effective potential. Quantum completeness refers to free evolution, corresponding to $\mathcal{P}[\Phi]=$ $\sqrt{-g_{t t}} / 2 g_{\Sigma}(\mathrm{d} \Phi, \mathrm{d} \Phi)$, possibly supplemented by a mass term. The wave functional of the ground state evolves from the initial Cauchy surface $\Sigma_{t_{0}}$ backwards in time to $\Sigma_{t}\left(t \in\left(0, t_{0}\right)\right)$ as $\Psi[\phi](t)=\mathcal{E}\left(t, t_{0}\right) \Psi[\phi]\left(t_{0}\right)$, with

$$
\mathcal{E}\left(t, t_{0}\right)=\exp \left(-\mathrm{i} \int_{t_{0}}^{t} \mathrm{~d} t^{\prime} \int_{\Sigma_{t^{\prime}}} \mathrm{d} \mu_{z} \mathcal{H}[\Phi]\right),
$$

where $\mathrm{d} \mu_{z}$ denotes the covariant volume form with respect to $g_{\Sigma}$, and $z$ refers to the coordinate neighbourhood. In $\mathcal{B}$, the evolution operator $\mathcal{E}$ is not unitary. Quantum completeness of $\mathcal{B}$ with respect to $(\mathcal{H}, \Phi)$ requires $\|\Psi[\phi]\|(t) \leq\|\Psi[\phi]\|\left(t_{0}\right) \forall t \in\left(0, t_{0}\right)$, implying that $\mathcal{B}$ can be a sink for probability but not a source, given it is not resolved in dynamical degrees of freedom.

We expect that a quadratic functional $\mathcal{K}_{2}[\phi, \phi](t)$ exists such that the wave functional $\Psi^{(0)}[\phi](t)$, corresponding to the ground state of $(\mathcal{H}, \Phi)$, is given by

$$
\Psi^{(0)}[\phi](t)=N^{(0)}(t) \exp \left(-\mathcal{K}_{2}[\phi, \phi](t)\right),
$$

with $N^{(0)}$ denoting the time-dependent normalisation, and $\mathcal{K}_{2}$ can be expressed in terms of the bi-local kernel function $K_{2}$ as

$$
\mathcal{K}_{2}[\phi, \phi](t)=\frac{1}{2} \int_{\Sigma_{t}} \mathrm{~d} \mu_{z_{1}} \mathrm{~d} \mu_{z_{2}} \phi\left(z_{1}\right) K_{2}\left(z_{1}, z_{2}, t\right) \phi\left(z_{2}\right)(5)
$$

In the vicinity of the Schwarzschild singularity the evolution simplifies considerably,

$$
\mathcal{E}\left(t, t_{0}\right) \rightarrow \exp \left(-\frac{\mathrm{i} c\left(t_{0}\right)}{4 M} \ln t \int_{\Sigma_{t_{0}}} \mathrm{~d} \mu_{z} \frac{1}{\sin ^{2} \theta} \frac{\delta^{2}}{\delta \phi^{2}(z)}\right),
$$

where $c\left(t_{0}\right)$ is a constant of integration. As a consequence, the kernel function becomes a contact term in this limit, $K_{2}\left(z_{1}, z_{2}, t\right) \rightarrow k_{2}(t) \delta^{(3)}\left(z_{1}, z_{2}\right)$, which is consistent with the conjecture by Belinskii, Khalatnikov and 
Lifshitz [9]: Close to a space-like singularity, the variation of observables on $\Sigma_{t}$ from one location to another becomes irrelevant compared to changes in time. Subleading corrections to the asymptotic form of $K_{2}$ deviate from a contact contribution without changing the qualitative result.

In leading order, the evolution of the wave functional is given by

$$
\lim _{t \rightarrow 0} \Psi^{(0)}[\phi](t)=\lim _{t \rightarrow 0}\left|\ln \left(t / t_{0}\right)\right|^{-\Lambda v\left(\Sigma_{t}\right) / 2}
$$

up to constant and phase factors, which are irrelevant for the analysis presented here. In (7), an ultra-violet cut-off $\Lambda$ and a volume regularisation $v\left(\Sigma_{t}\right)$ have been introduced. Clearly, the limit $t \rightarrow 0$ is not affected by this simple choice. Hence, the wave functional has vanishing support towards the Schwarzschild singularity, and $\left\|\Psi^{(0)}[\phi]\right\|(t) \rightarrow 0 \leq\left\|\Psi^{(0)}[\phi]\right\|\left(t_{0}\right)$ for $t \rightarrow 0$, as required for $\mathcal{B}$ to be quantum complete with respect to the dynamical system $(\mathcal{H}, \Phi)$.

Concerning an interpretation: Consider the set of observables $\mathcal{A}_{\Sigma_{t}}$ of $(\mathcal{H}, \Phi)$ localised on $\Sigma_{t}$. Following the logic of geodesic incompleteness, it could be expected that an observable $\mathcal{O}_{\Sigma_{t}}$ exists with an expectation $\left\langle\mathcal{O}_{\Sigma_{t}}\right\rangle_{\Psi^{(0)}}$ in the ground state that is ill-defined. However, this is not the case since the asymptotic surface $\Sigma_{0}$ does not support any population of fields $\phi$, because the associated probability measure vanishes there.

As an example, consider a free field theory $(\mathcal{H}, \Phi)$ in the ground state described by the Schrödinger wavefunctional $\Psi^{(0)}[\phi](t)$, where $\phi$ denotes a classical field configuration over the hypersurface $\Sigma_{t}, t \in(0,2 M)$. Introducing an auxiliary source $\mathcal{J}$ coupling by $\Psi_{\mathcal{J}}^{(0)}[\phi](t):=$ $\Psi^{(0)}[\phi](t) \exp (\mathcal{J}[\phi](t))$ facilitates the description of measurement processes. Observables are evaluated in the ground state $|\Omega\rangle^{\mathcal{J}}$ in the presence of the auxiliary source, which is subsequently set to zero. Compositions of the configuration operator are then replaced by the corresponding succession of derivations $\delta_{\phi}$, where

$$
\delta_{\phi}:=\int_{\Sigma_{t}} \mathrm{~d} \mu_{x} \frac{\phi(x)}{\sqrt{\operatorname{det}\left(g_{\Sigma}\right)}} \frac{\delta}{\delta J(x)}
$$

is a directional derivative in field space, with $J$ denoting the ultra-local representation of $\mathcal{J}$. For instance, in the presence of an auxiliary source

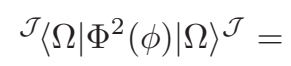

$$
\begin{aligned}
& \delta_{\phi}^{2} \exp \left\{\frac{1}{4} \frac{1}{\sqrt{\operatorname{det}\left(g_{\Sigma}\right)}}\left[\operatorname{Re}\left(\mathcal{K}_{2}\right)\right]^{-1}[J, J]\right\} \mathcal{W}^{(0)}(t),
\end{aligned}
$$

where $\mathcal{W}^{(0)}(t):=\left\|\Psi^{(0)}[\phi]\right\|^{2}(t)$. In the absence of the auxiliary source, the ground-state expectation becomes

$$
\begin{aligned}
& \left\langle\Omega\left|\Phi^{2}(\phi)\right| \Omega\right\rangle= \\
& \frac{1}{2} \sqrt{\operatorname{det}\left(g_{\Sigma}\right)}\left[\operatorname{Re}\left(\mathcal{K}_{2}\right)\right]^{-1}[\phi, \phi] \mathcal{W}^{(0)}(t),
\end{aligned}
$$

which is real and semi-positive definite. In particular, in the vicinity of the limiting hypersurface $\Sigma_{0}$, the groundstate expectation approaches zero, due to the temporal support properties associated with the probability density. Similarly, it can be shown that $\left\langle\Omega\left|\pi^{2}(\phi)\right| \Omega\right\rangle$ is real and semi-positive definite, and approaches zero towards $\Sigma_{0}$. Therefore, the ground-state expectation of $\mathcal{H}$ is in $\mathbb{R}^{+}$and vanishes towards the would-be singular hypersurface $\Sigma_{0}$.

\section{SELF-INTERACTIONS}

In this section, polynomial self-interactions are included and their impact on the stability of the ground state is analysed. For definiteness we consider the effective potential $\mathcal{P}_{\text {int }}[\Phi]:=\mathcal{P}[\Phi]+\sqrt{-g_{t t}} \lambda \Phi^{4} / 4$ !. The dimensionless coupling $\lambda$ is chosen such that perturbation theory is applicable in a neighbourhood of $\Sigma_{t_{0}}$. Self-interactions deform the ground-state wave functional away from its Gaussian shape

$$
\Psi_{\text {int }}^{(0)}[\phi](t)=\Psi^{(0)}[\phi](t) \times \exp (\lambda \mathcal{D}[\phi](t)) .
$$

The deformation functional $\mathcal{D}=\mathcal{D}_{2}+\mathcal{D}_{4}$ is a sum of the time-dependent nonlinear functionals $\mathcal{D}_{2}: \mathcal{S}^{\otimes 2} \rightarrow \mathcal{C}\left(\mathbb{R}^{+}\right)$ and $\mathcal{D}_{4}: \mathcal{S}^{\otimes 4} \rightarrow \mathcal{C}\left(\mathbb{R}^{+}\right.$), where $\mathcal{S}$ denotes the field space and $\mathcal{C}\left(\mathbb{R}^{+}\right)$is the space of functions depending smoothly on time. As before, local versions can be introduced via kernel functions $D_{2}$ and $D_{4}$, respectively. Close to the singularity, $D_{j}=d_{j}(t) \Pi_{a=1}^{j} \delta^{(3)}\left(z_{a}\right), j \in\{2,4\}$, i.e. any spatial information close to $\Sigma_{0}$ is concentrated in a single event. Again, only the temporal gradients matter. In this limit, the kernel functions obey the coupled kernel equations $\mathrm{i} \partial_{t} \underline{d}=\sqrt{\operatorname{det}(g)} \mathfrak{a} \underline{d}$, where $\underline{d}:=\left(d_{2}, d_{4}\right)^{\mathrm{T}}$ and $\mathfrak{a}$ is a two-by-two matrix with coefficients $\mathfrak{a}_{11}=k(t)$, $\mathfrak{a}_{12}=1, \mathfrak{a}_{21}=0$ and $\mathfrak{a}_{22}=k(t)$. The asymptotic solution is $\underline{d}(t)=(1,1)^{\mathrm{T}} /|\ln (t)| \rightarrow 0$ for $t \rightarrow 0$.

As a consequence, deformations of the Gaussian ground state, induced by self-interactions, become less and less important towards the black-hole singularity, $\mathcal{D}[\phi](t) \rightarrow 0$ for $t \rightarrow 0$. In greater detail, asymptotically $\mathcal{D}_{j} \propto t^{3 j / 2} /|\ln (t)|$ for $j \in\{2,4\}$ and, hence,

$$
\lim _{t \rightarrow 0} \Psi_{\text {int }}^{(0)}[\phi](t)=\lim _{t \rightarrow 0} \Psi^{(0)}[\phi](t)=0 .
$$

Thus, close to $\Sigma_{0}$ (i.e. for Schwarzschild times $t \ll t_{0}$ ), the dynamical systems $(\mathcal{H}, \Phi)$ and $\left(\mathcal{H}_{\pi}, \Phi\right)$ may be identified.

This proves that self-interactions cannot cure the classical black-hole singularity via back-reaction effects on the external geometry. Close to the singularity selfinteractions loose their impact on the evolution of the system. The system becomes asymptotically free, and the stability requirement on the quantum theory is too stringent to allow the free theory to destabilise even towards $\Sigma_{0}$. Hence, the quantum fields are totally ignorant about the singularity. From this point of view the classical singularity needs no resolution since it appears as a mathematical artefact with no observational consequences whatsoever, assuming the measurements are anchored in the framework provided by quantum theory. 
It seems that quantum completeness of the Schwarzschild black-hole protects general relativity against its classical incompleteness. In fact, the potential harmful implications associated with $\Sigma_{0}$ decouple from quantum measurements.

A more abstract reasoning is the following: Consider classical fields $\phi$ as configurations in $\mathcal{C}^{2}(\Sigma)$. In order to ensure a probabilistic interpretation, the Schrödinger wave-functionals have to be normalisable with respect to some functional measure $\mathrm{D} \phi$. Wave functionals enjoying this property can be collected in a state space $\mathcal{L}^{2}\left(\mathcal{C}^{2}(\Sigma), \mathrm{D} \phi\right)$, which obviously requires a mathematical justification beyond the scope of this article. Even for these wave functionals, $\mathcal{H}_{\pi}$ is not self-adjoint, but the spectrum contains only functions with a positivesemidefinite imaginary part. As a consequence, towards the singularity $\mathcal{E}\left(t, t_{0}\right)$ becomes exponentially damped. Self-interactions cannot harm this regularisation of the classical singularity, simply because they are given as compositions of multiplication operators. Furthermore, $\mathcal{H} \rightarrow \mathcal{H}_{\pi}$ towards the singularity, where the limit is taken in a generalisation of the strong operator topology appropriate for the functional calculus involved here. From this point of view, a self-interacting quantum probe is totally ignorant about the classical singularity.

\section{EXCITATIONS}

Excitations of the ground state are not an integral component in the definition of quantum completeness. If the dynamical system $(\mathcal{H}, \Phi)$ is unstable, then excitations might trigger a transition towards a stable ground state.

The ground state is an eigenstate of the conjugated momentum field, $\left(\pi(t, x)-\mathrm{i} \delta \mathcal{K}_{2} / \delta \phi(t, x)\right) \Psi^{(0)}[\phi](t)=0$, and a kernel element of the operator valued functional $a[f](t)$, describing the absorption of a field $f \in \mathcal{S}_{\text {os }}$, where the index 'os' implies the restriction to on-shell fields. The above eigenstate equation is a ultra-local version of absorption. Emission can be considered accordingly using the adjoint $a^{\dagger}[f](t)$. As usual, on $\Sigma_{t}$ the following algebraic relation holds:

$$
\left[a[f](t), a^{\dagger}\left[f^{\prime}\right](t)\right]=2 \operatorname{Re}\left(\mathcal{K}_{2}\left[f, f^{\prime}\right]\right)(t) .
$$

An excitation relative to the ground state is given by $\Psi^{(1)}[f, \phi](t):=a^{\dagger}[f](t) \Psi^{(0)}[\phi](t)$. Note that $\phi \in \mathcal{S}$, while $f \in \mathcal{S}_{\mathrm{os}} \subset \mathcal{S}$, i.e. the emission operator creates on-shell information and stores it in the excited state $\Psi^{(1)}[f, \phi](t)=2 \operatorname{Re}\left(\mathcal{K}_{2}[f, \phi]\right)(t) \Psi^{(0)}[\phi](t)$. Therefore, exciting the ground state by emitting an on-shell quantum simply results in a functional renormalisation of the ground state. Owing to the algebraic relation (13), we find

$$
\left\|\Psi^{(1)}\right\|^{2}(t)=2 \operatorname{Re}\left(\mathcal{K}_{2}[f, f]\right)(t)\left\|\Psi^{(0)}\right\|^{2}(t) .
$$

So quantum completeness of the ground state is a necessary but not sufficient criterion for the stability of the first excited state. In addition, $\operatorname{Re}\left(\mathcal{K}_{2}[f, f]\right)(t)<\infty$ is required for all $f \in \mathcal{S}_{\text {os }}$.

For vanishing Schwarzschild time, the renormalisation becomes constant and is therefore inconsequential. This can be seen as follows: Up to sub-leading contributions, the time dependence of $f(t, x)=T(t) R(x)$ is given by $\left(t \partial_{t}^{2}+\partial_{t}\right) T=\kappa T$ with $\kappa$ a constant determined by the equation for $R$. For vanishing Schwarzschild time, $T$ should be singular. Introducing $\tau:=\zeta t$, and taking the limit $t \rightarrow 0, \zeta \rightarrow \infty$ such that the rescaled Schwarzschild time $\tau$ remains constant, the equation of motion for $T$ becomes $\left(\tau \partial_{\tau}^{2}+\partial_{\tau}\right) T=0$. Thus, up to an additive constant, $T=\ln (t)$. Therefore, $\operatorname{Re}\left(\mathcal{K}_{2}\left[f, f^{\prime}\right]\right)=$ const, because the time dependence of the corresponding kernel function cancels exactly against the time dependence of the mode functions and the volume form. Note that the additive constant poses no problem due to the prescription for taking the asymptotic limit. As a consequence, $\left\|\Psi^{(1)}\right\|^{2} \rightarrow 0$ towards the black-hole singularity. In fact, as can be seen by induction, all excitations $\Psi^{(n)}(n \in \mathbb{N})$ give rise to a vanishing probability measure on $\Sigma_{0}$. Neither the ground state nor any excited states are populated with fields on $\Sigma_{0}$. The natural probability measure protects the stability of any state, and this stability protection can be traced back to a persistent ground state.

\section{DISCUSSION}

In this article the notion of quantum mechanical completeness is adapted to situations where the only adequate description is in terms of (interacting) quantum fields in dynamical space-times. The adaption necessarily generalises from requiring a unitary evolution by demanding a normalisation condition that ensures a probabilistic interpretation. Of course, this condition reduces to unitarity in the absence of dynamical sources. While originally stated for free fields in a Gaussian groundstate, it is shown to extend to interacting quantum fields in arbitrary states. It is tempting to expect that this extension is rather trivial if the ground state admits a Gaussian wave functional. This expectation has to be confronted with the dynamics of the external space-time that sources different terms in the Hamiltonian differently. It is important to stress that both, geodesic and quantum completeness, assume a background space-time, which is either diagnosed by test particles or by test fields, respectively. This assumption, however, can only be investigated in a quantum theory of fields.

Whether a given dynamics is consistent with a probabilistic interpretation is usually examined in an asymptotic framework pertinent to scattering theory. There the stability of the ground state is studied in the presence of an external source after an infinite amount of time has passed. This is clearly not an option in arbitrary space-times. Furthermore, it seems intuitive that stability challenges are anchored in the vicinity of spacetime singularities, which suggests a more local stability 
analyses. For these reasons, the Schrödinger representation of quantum field theory is quite convenient, which allows, in particular, to investigate the stability of a given quantum system in a dynamical space-time after a finite amount of time elapsed.

The Schrödinger representation requires a functional generalisation of many quantum-mechanical concepts. In particular, choosing the configuration field as the multiplication operator, the associated momentum field becomes a functional derivative. And the norm of a wave functional requires a functional integral over the configuration fields. Many of these functional techniques can be disputed on mathematical grounds. However, the stability analysis is entirely at the qualitative level and not based on any specific regularisation.

The main result of this article is that Schwarzschild black-holes are quantum complete, which has a very precise meaning. However, equally precise they are qualified as geodesically incomplete space-times by the singularity theorem of Hawking and Penrose. Of course, both completeness notions are logically consistent within their respective domains of validity. If we are to derive further consequences from these notions, in particular concerning the consistency of black holes and of general relativity, it is important to understand which domain and therefore which completeness notion is applicable given the physical conditions. Our point of view advocated here is the following: Geodesic completeness is a concept in the category of smooth manifolds as models for space-times. To the extent that we can be certain that these models can be probed by physical events it is falsifiable. In the vicinity of space-like singularities, spatial correlations become trivial, i.e. events can only be spatially correlated if they are stacked on top of each other. As might be expected, what matters in the vicinity of a space-like singularity are temporal correlations. In fact, temporal gradients correspond to a characteristic length scale that is smaller than the length scale characterising the spatial extent of any conceivable classical measurement device. Therefore, any completeness diagnosis based on classical measurements is inappropriate given the physical conditions. Any measurement process in the vicinity of a black-hole singularity has to rely on quantum field theory. In the context of classical singularity theorems, the only falsifiable completeness notion applicable to blackhole interiors is quantum completeness.

This argument is not in conflict with the logic underlying the usual quantisation prescription, precisely because the probability measure is always well-defined. In par- ticular, the Gaussian ground-state is respected by selfinteractions, provided the system was in a weak-coupling regime initially. This is in accordance with the intuitive expectation that the free dynamics (temporal correlations) dominates in the vicinity of the singularity. Consequently, excitations relative to the ground state cannot change the conclusion. Let us stress that these results are in full accordance with the dynamical stability of classical field configuration in Schwarzschild space-time, as has been established in [10 12]. Temporal support for field configurations is strictly restricted to the interval $\left(0, t_{0}\right]$ with the initial time $t_{0}<r_{\mathrm{g}}$, and the field configurations are smooth on this interval.

Black-hole interiors are quantum complete and this notion is sensible from a physics point of view even in the vicinity of the classically singular hypersurface. In contrast, geodesic incompleteness of black holes, albeit a mathematical rigorous qualification, is not a physical statement since any operative measurement has to employ physics beyond point particle dynamics. As a consequence, the classically singular hypersurface bears no impact on observables based on bookkeeping devices (fields) with sensible dynamics. Less sensible is the argument that geometrical observables such as the Kretschmann scalar would diverge at the origin. This line of argument is already invalidated for simple bound-state problems in quantum mechanics, for instance the hydrogen atom. Clearly, the Coulomb potential enjoys geodesic and potential incompleteness, which is inconsequential for hydrogen as a quantum bound-state. Albeit the singular structure in this case is just a point, quantum completeness is established by arguments related to the support properties of the probability measure, as well. In the case of black holes, the singular structure is space-like, but corresponds to a limiting instant in time.

\section{ACKNOWLEDGMENTS}

It is a great pleasure to thank Ingemar Bengtsson, Kristina Giesel, Maximilian Kögler, Florian Niedermann, and Maximilian Urban for delightful discussions. We thank Robert C. Myers for sharing his ideas about this topic. We appreciate financial support of our work by the DFG cluster of excellence 'Origin and Structure of the Universe', the Humboldt Foundation, and by TRR 33 'The Dark Universe'.
[1] S. W. Hawking and R. Penrose, Proceedings of the Royal Society of London A 314, 529 (1970).

[2] M. Reed and B. Simon, Methods of modern mathematical physics II: Fourier Analysis, Self-Adjointness, Vol. 2 (Elsevier, 1975).

[3] G. Horowitz and D. Marolf, Physical Review D 52, 5670
(1995).

[4] A. Ashtekar and A. Magnon, in Proceedings of the Royal Society of London A: Mathematical, Physical and Engineering Sciences, Vol. 346 (The Royal Society, 1975) pp. 375-394.

[5] R. M. Wald, Journal of Mathematical Physics 20 (1978). 
[6] This corresponds to relaxing the requirements posed on evolution generators to linear unbounded operators that are closed, have a dense domain, whose resolvent set contains all positive reals and whose resolvent operator, which can be thought of as the Laplace transform of the semigroup, is bounded from above for each element of the resolvent set by its inverse.

[7] S. Hofmann and M. Schneider,
Phys. Rev. D 91, 125028 (2015)

[8] J. Ehlers and W. Kundt, in The Theory of Gravitation (John Wiley \& Sons, Inc., 1962) pp. 49-101.

[9] V. Belinskii, I. Khalatnikov, and E. Lifshitz, Advances in Physics 19, 525 (1970)

[10] B. P. Jensen and P. Candelas, Phys. Rev. D 33, 1590 (1986)

[11] E. Elizalde, Phys. Rev. D 36, 1269 (1987)

[12] E. Elizalde, Phys. Rev. D 37, 2127 (1988) 